\title{
Robust State Feedback Stabilization of Uncertain Discrete-Time Switched Linear Systems Subject to Actuator Saturation
}

\author{
Xinquan Zhang, ${ }^{1}$ Guoliang Wang, ${ }^{1}$ and Jun Zhao ${ }^{2}$ \\ ${ }^{1}$ School of Information and Control Engineering, Liaoning Shihua University, Fushun 113001, China \\ ${ }^{2}$ State Key Laboratory of Synthetical Automation for Process Industries, Northeastern University, Shenyang 110819, China \\ Correspondence should be addressed to Xinquan Zhang; zxq_19800126@163.com
}

Received 16 September 2014; Accepted 12 January 2015

Academic Editor: Seenith Sivasundaram

Copyright (c) 2015 Xinquan Zhang et al. This is an open access article distributed under the Creative Commons Attribution License, which permits unrestricted use, distribution, and reproduction in any medium, provided the original work is properly cited.

\begin{abstract}
The robust stabilization problem is investigated for a class of discrete-time switched linear systems with time-varying normbounded uncertainties and saturating actuators by using the multiple Lyapunov functions method. A switching law and a state feedback law are designed to asymptotically stabilize the system with a large domain of attraction. Based on the multiple Lyapunov functions method, sufficient conditions are obtained for robust stabilization. Furthermore, when some parameters are given in advance, the state feedback controllers and the estimation of domain of attraction are presented by solving a convex optimization problem subject to a set of linear matrix inequalities (LMI) constraints. A numerical example is given to show the effectiveness of the proposed technique.
\end{abstract}

\section{Introduction}

Switched systems are an important class of hybrid dynamic systems, which consist of a family of continuous-time or discrete-time subsystems and a switching law orchestrating which subsystem to be activated during a certain interval of time. In the last decade, the study of switched systems has received a growing attention in control theory $[1-5]$ and practice [6-9]. The motivation for studying switched systems stems from the fact that many real-world processes and systems can be modeled as switched systems, including chemical processes, computer disk drives, network control, power systems, communication industries, robotic manufacture, and multiple-model systems. A lot of attention is focused on the stability issue of switched systems $[1,2]$, because stability is the most delicate and important issue in the study. Many analytical approaches and techniques have been developed for this issue, for example, common Lyapunov function approach [10-12], switched Lyapunov function approach [13], multiple Lyapunov functions approach [14-16], and the average dwell-time method [17]. For the switched linear systems, the multiple Lyapunov functions method has been considered largely as one of the most effective tools.
It is well known that nearly all control systems in practice often operate with actuator saturation, since all control devices are limited by inherent physical constraints. Saturation is a source of degradation of system performance, occurrence of limit cycle, more than one equilibrium states some of which may have deterrent stability behavior, and even system instability caused by some large perturbation. Hence, the stability analysis and synthesis for nonswitched control systems subject to actuator saturation nonlinearities have been receiving increased attention for years and decades due to the practical and theoretical importance, which may also be seen in the recent works [18-22]. It is pointed out in $[20,22]$ that there are two main approaches dealing with actuator saturation nonlinearities. The first strategy is to neglect actuator saturation and design a linear controller that meets the performance specifications in the first stage of the control design process and then add an antiwindup compensator to minimize the influence of saturation [20]. The second strategy is to take actuator saturation into account at the outset of the control design and then design a linear controller which guarantees the asymptotic stability of the system [22]. The main goal in these two strategies is to obtain 
a larger estimate of the domain of attraction, despite the presence of saturation.

For switched systems with actuator saturation, the analysis and synthesis become more difficult owing to the phenomena of interacting switching and actuator saturation nonlinearities. In turn, the results for switched systems with actuator saturation are very few. Via utilizing multiple Lyapunov functions method, [23] considered the design of switching scheme for a class of switched linear systems in the presence of actuator saturation. In [24], a switching antiwindup design was proposed for a linear system subject to actuator saturation, which is based on the min-function of multiple quadratic Lyapunov functions. Robust stabilization problem for a class of switched linear systems with saturating actuators and uncertainties was investigated in [25] also via the multiple Lyapunov functions method. These papers are devoted to the continuous-time switched systems subject to input saturation. Reference [26] investigated the stabilization problem for a class of discrete-time linear switched systems subject to actuator saturations via a composite Lyapunov function method. References [27, 28] extended the result in [26] by showing that the same conditions also allow that the union of all the corresponding level sets of functions constitutes a large region of asymptotic stability of the switched system. All the results mentioned above are about the stabilization problem of discrete-time linear switched systems under arbitrary switchings. To the best of the authors' awareness, for uncertain discrete-time switched linear systems subject to actuator saturation no results on the problem of the robust stabilization with resort to the multiple Lyapunov functions method have been reported in the existing literature, which motivates the present study.

This paper studies the problems of robust stability analysis and robustly stabilizing control synthesis for a class of uncertain discrete-time switched linear systems subject to actuator saturation via multiple Lyapunov functions method. Sufficient conditions of asymptotic stability are obtained. Furthermore, the problem of designing linear state feedback controllers which guarantee the stability of the closed-loop system and a larger estimation of domain of attraction is formulated and solved in terms of the solution of a set of linear matrix inequalities (LMIs).

Compared with the existing results for switched systems subject to actuator saturation, there are two distinct features. Firstly, the multiple Lyapunov functions method is used to consider the stability problem of discrete-time switched systems with input saturation for the first time and this problem does not require solvability for every subsystem, while in the existing literature, this problem has been studied by using the switched Lyapunov function method and the solvability of the problem for every subsystem is required; secondly, controllers are designed based on the multiple Lyapunov functions method to achieve better performance (such as large the estimation of domain of attraction) for discrete-time switched systems with input saturation, while the existing literature either only focuses on the analysis, not design, or investigates the design issue that uses the switched Lyapunov function and dwell time methods.
Further this paper is organized as follows: Section 2 introduces the problem formulation. Section 3 presents the main results which consist of the stability analysis, the controller design, and the estimation of domain of attraction. An example is shown in Section 4 to illustrate the feasibility of our results. Finally, conclusions end the paper in Section 5.

\section{Problem Statement and Preliminaries}

We consider the following class of uncertain discrete-time switched linear systems with input saturation:

$$
\begin{aligned}
x(k+1)= & \left(A_{\sigma(k)}+\Delta A_{\sigma(k)}\right) x(k) \\
& +\left(B_{\sigma(k)}+\Delta B_{\sigma(k)}\right) \operatorname{sat}(u(k)),
\end{aligned}
$$

where $k \in Z^{+}, x(k) \in R^{n}$ is the state vector, and $u(k) \in R^{m}$ is the control input vector. The function sat : $R^{m} \rightarrow R^{m}$ is the vector valued standard saturation function defined as

$$
\begin{aligned}
& \operatorname{sat}(u)=\left[\operatorname{sat}\left(u^{1}\right), \ldots, \operatorname{sat}\left(u^{m}\right)\right]^{T}, \\
& \operatorname{sat}\left(u^{j}\right)=\operatorname{sign}\left(u^{j}\right) \min \left\{1,\left|u^{j}\right|\right\}, \quad j \in Q_{m}=\{1, \ldots, m\} .
\end{aligned}
$$

The function $\sigma(k): Z^{+} \rightarrow I_{N}=\{1, \ldots, N\}$ is a piecewise constant switching signal; $\sigma(k)=i$ means that the $i$ th subsystem is active. $A_{i}, B_{i}$ are constant matrices of appropriate dimensions that describe the set of nominal systems. $\Delta A_{i}$, $\Delta B_{i}$ denote unknown matrices with time-varying parameter uncertainties appearing in system matrices and having the following form:

$$
\left[\Delta A_{i}, \Delta B_{i}\right]=T_{i} \Gamma(k)\left[F_{1 i}, F_{2 i}\right], \quad i \in I_{N},
$$

where $T_{i}, F_{1 i}$, and $F_{2 i}$ are given constant matrices with proper dimensions which characterize the structure of uncertainties. Matrix $\Gamma(k)$ denotes the unknown time-varying matrix function satisfying

$$
\Gamma^{T}(k) \Gamma(k) \leq I
$$

In this paper, we consider the following linear state feedback control laws:

$$
u(k)=F_{i} x(k), \quad i \in I_{N},
$$

where $F_{i}$ are matrices either given beforehand or are to be designed. Then the closed-loop system is

$$
x(k+1)=\left(A_{i}+\Delta A_{i}\right) x(k)+\left(B_{i}+\Delta B_{i}\right) \operatorname{sat}\left(F_{i} x(k)\right) .
$$
results.

The following lemmas will be used to develop the main

Lemma 1 (see [29]). Let $Y, U, V$ be given matrices of appropriate dimensions; then for any matrix $\Gamma$ satisfying $\Gamma^{T} \Gamma \leq$ I,

$$
Y+U \Gamma V+V^{T} \Gamma^{T} U^{T}<0
$$

if and only if there exists a constant $\lambda>0$ such that

$$
Y+\lambda U U^{T}+\lambda^{-1} V^{T} V<0 .
$$


For a positive definite matrix $P \in R^{n \times n}$ and a scalar $\rho>0$, an ellipsoid $\Omega(P, \rho)$ is defined as

$$
\Omega(P, \rho)=\left\{x \in R^{n}: x^{T} P x \leq \rho\right\} .
$$

Let $F^{j}$ be the $j$ th row of the matrix $F \in R^{m \times n}$. We define the symmetric polyhedron

$$
L(F)=\left\{x \in R^{n}:\left|F^{j} x\right| \leq 1, j \in Q_{m}\right\} .
$$

Let $D$ be the set of $m \times m$ diagonal matrices whose diagonal elements are either 1 or 0 . For example, if $m=2$, then

$$
D=\left\{\left[\begin{array}{ll}
1 & 0 \\
0 & 1
\end{array}\right],\left[\begin{array}{ll}
1 & 0 \\
0 & 0
\end{array}\right],\left[\begin{array}{ll}
0 & 0 \\
0 & 1
\end{array}\right],\left[\begin{array}{ll}
0 & 0 \\
0 & 0
\end{array}\right]\right\}
$$

There are $2^{m}$ elements in $D$. Suppose that each element of $D$ is labeled as $D_{s}, s \in Q=\left\{1,2, \ldots, 2^{m}\right\}$, and denote $D_{s}^{-}=I-D_{s}$. Clearly, $D_{s}^{-}$is also an element of $D$ if $D_{s} \in D$.

Lemma 2 (see [22]). Let $F, H \in R^{m \times n}$ be given. For any $x \in$ $R^{n}$, if $x \in L(H)$, then

$$
\operatorname{sat}(F x) \in \operatorname{co}\left\{D_{s} F x+D_{s}^{-} H x, s \in Q\right\},
$$

where co\{r\} denotes the convex hull of a set. Consequently, $\operatorname{sat}(F x)$ can be expressed as

$$
\operatorname{sat}(F x)=\sum_{s=1}^{2^{m}} \eta_{s}\left(D_{s} F+D_{s}^{-} H\right) x,
$$

where $\sum_{s=1}^{2^{m}} \eta_{s}=1,0 \leq \eta_{s} \leq 1$. We note that the parameters $\eta_{s}$ in (13) are functions of the state $x$.

Lemma 3 (Schur's complements). Given the symmetric matrix $A=\left[\begin{array}{ll}A_{11} & A_{12} \\ A_{12}^{T} & A_{22}\end{array}\right]$, the following statements are equivalent:

(1) $A<0$;

(2) $A_{11}<0, A_{22}-A_{12}^{T} A_{11}^{-1} A_{12}<0$;

(3) $A_{22}<0, A_{11}-A_{12} A_{22}^{-1} A_{12}^{T}<0$.

The objective of this paper is to design a switching law and a state feedback law of the form (5) such that the resulting closedloop system (6) is locally asymptotically stable at the origin of the state space with a domain of attraction as large as possible.

\section{Main Results}

In this section, we will develop stability conditions and design stabilizing controllers.

3.1. Stability Analysis. In this subsection, we assume the control laws $u_{i}=F_{i} x$ are known beforehand. Then, we give a sufficient condition for stability of switched system (6) by means of the multiple Lyapunov functions method.
Theorem 4. Suppose that there exist positive definite matrices $P_{i}$, matrices $H_{i}$, and a set of scalars $\beta_{i r} \geq 0, \lambda_{i}>0$ such that

$$
\left[\begin{array}{cccc}
-P_{i}+\sum_{r=1, r \neq i}^{N} \beta_{i r}\left(P_{r}-P_{i}\right) & * & * & * \\
P_{i}\left[A_{i}+B_{i}\left(D_{s} F_{i}+D_{s}^{-} H_{i}\right)\right] & -P_{i} & * & * \\
0 & T_{i}^{T} P_{i} & -\lambda_{i}^{-1} I & * \\
F_{1 i}+F_{2 i}\left(D_{s} F_{i}+D_{s}^{-} H_{i}\right) & 0 & 0 & -\lambda_{i} I
\end{array}\right]<0,
$$

Moreover, assume $\Omega\left(P_{i}, 1\right) \cap \Phi_{i} \subset L\left(H_{i}\right)$. Then, the switched system (6) is robustly asymptotically stable at the origin with $\cup_{i=1}^{N}\left(\Omega\left(P_{i}, 1\right) \cap \Phi_{i}\right)$ contained inside the domain of attraction under the state dependent switching law

$$
\sigma(k)=\operatorname{argmin}\left\{x^{T}(k) P_{i} x(k), i \in I_{N}\right\},
$$

where $\Phi_{i}=\left\{x(k) \in R^{n}: x^{T}(k)\left(P_{r}-P_{i}\right) x(k) \geq 0, \forall r \in I_{N}, r \neq\right.$ i\}.

Proof. By virtue of Lemma 2, for every $x(k) \in \Omega\left(P_{i}, 1\right) \cap \Phi_{i} \subset$ $L\left(H_{i}\right)$,

$$
\operatorname{sat}\left(F_{i} x(k)\right) \in \operatorname{co}\left\{D_{s} F_{i} x(k)+D_{s}^{-} H_{i} x(k), s \in Q\right\} .
$$

It follows that

$$
\begin{aligned}
\left(A_{i}+\Delta A_{i}\right) x(k)+\left(B_{i}+\Delta B_{i}\right) \operatorname{sat}\left(F_{i} x(k)\right) \\
\in \operatorname{co}\left\{\left(A_{i}+\Delta A_{i}\right) x(k)+\left(B_{i}+\Delta B_{i}\right)\right. \\
\left.\cdot\left(D_{s} F_{i} x(k)+D_{s}^{-} H_{i} x(k)\right), s \in Q\right\} .
\end{aligned}
$$

In view of the switching law (15), for $\forall x(k) \in \Omega\left(P_{i}, 1\right) \cap$ $\Phi_{i} \subset L\left(H_{i}\right)$, the $i$ th subsystem is active.

Choose the Lyapunov function candidate for system (6) as

$$
V(x(k))=V_{\sigma(k)}(x(k))=x^{T}(k) P_{\sigma(k)} x(k) .
$$

We split the proof into two parts.

Case 1. When $\sigma(k+1)=\sigma(k)=i$, for $\forall x(k) \in \Omega\left(P_{i}, 1\right) \cap \Phi_{i} \subset$ $L\left(H_{i}\right)$, it follows that

$$
\begin{aligned}
\Delta V(x(k))= & V_{i}(x(k+1))-V_{i}(x(k)) \\
= & x^{T}(k+1) P_{i} x(k+1)-x^{T}(k) P_{i} x(k) \\
\leq & \max _{s \in Q} x^{T}(k)\left[\left(A_{i}+\Delta A_{i}\right)+\left(B_{i}+\Delta B_{i}\right)\right. \\
\left.\cdot\left(D_{s} F_{i}+D_{s}^{-} H_{i}\right)\right]^{T} & \\
& \quad P_{i}\left[\left(A_{i}+\Delta A_{i}\right)+\left(B_{i}+\Delta B_{i}\right)\right. \\
& \left.\cdot\left(D_{s} F_{i}+D_{s}^{-} H_{i}\right)\right] x(k)-x^{T}(k) P_{i} x(k) .
\end{aligned}
$$


Case 2. When $\sigma(k)=i, \sigma(k+1)=r$, and $i \neq r$, for $\forall x(k) \epsilon$ $\Omega\left(P_{i}, 1\right) \cap \Phi_{i} \subset L\left(H_{i}\right)$, using switching law (15), we have

$$
\begin{aligned}
\Delta V(x(k)) & =x^{T}(k+1) P_{r} x(k+1)-x^{T}(k) P_{i} x(k) \\
& \leq x^{T}(k+1) P_{i} x(k+1)-x^{T}(k) P_{i} x(k) .
\end{aligned}
$$

From Cases 1 and 2, we obtain

$$
\begin{array}{r}
\Delta V(x(k)) \leq \max _{s \in \mathrm{Q}} x^{T}(k)\left[\left(A_{i}+\Delta A_{i}\right)+\left(B_{i}+\Delta B_{i}\right)\right. \\
\left.\cdot\left(D_{s} F_{i}+D_{s}^{-} H_{i}\right)\right]^{T} \\
\cdot P_{i}\left[\left(A_{i}+\Delta A_{i}\right)+\left(B_{i}+\Delta B_{i}\right)\right. \\
\left.\cdot\left(D_{s} F_{i}+D_{s}^{-} H_{i}\right)\right] x(k)-x^{T}(k) P_{i} x(k), \\
\forall x(k) \in \bigcup_{i=1}^{N}\left(\Omega\left(P_{i}, 1\right) \cap \Phi_{i}\right) .
\end{array}
$$

Then, by Lemma 3, (14) is equivalent to

$$
\begin{aligned}
& {\left[\begin{array}{cc}
-P_{i}+\sum_{r=1, r \neq i}^{N} \beta_{i r}\left(P_{r}-P_{i}\right) & * \\
P_{i}\left[A_{i}+B_{i}\left(D_{s} F_{i}+D_{s}^{-} H_{i}\right)\right] & -P_{i}
\end{array}\right]+\lambda_{i}\left[\begin{array}{c}
0 \\
P_{i} T_{i}
\end{array}\right]\left[\begin{array}{ll}
0 & T_{i}^{T} P_{i}
\end{array}\right]} \\
& +\lambda_{i}^{-1}\left[\begin{array}{c}
{\left[F_{1 i}+F_{2 i}\left(D_{s} F_{i}+D_{s}^{-} H_{i}\right)\right]^{T}} \\
0
\end{array}\right] \\
& \text { - }\left[F_{1 i}+F_{2 i}\left(D_{s} F_{i}+D_{s}^{-} H_{i}\right) 0\right]<0 \text {. }
\end{aligned}
$$

Thus, using Lemma 1 we have

$$
\left[\begin{array}{cc}
-P_{i}+\sum_{r=1, r \neq i}^{N} \beta_{i r}\left(P_{r}-P_{i}\right) & * \\
P_{i}\left[\left(A_{i}+\Delta A_{i}\right)+\left(B_{i}+\Delta B_{i}\right)\left(D_{s} F_{i}+D_{s}^{-} H_{i}\right)\right] & -P_{i}
\end{array}\right]
$$

$<0$.
Again, from the Lemma 3, we get

$$
\begin{aligned}
{\left[\left(A_{i}+\Delta A_{i}\right)+\left(B_{i}+\Delta B_{i}\right)\left(D_{s} F_{i}+D_{s}^{-} H_{i}\right)\right]^{T} } \\
\quad \cdot P_{i}\left[\left(A_{i}+\Delta A_{i}\right)+\left(B_{i}+\Delta B_{i}\right)\left(D_{s} F_{i}+D_{s}^{-} H_{i}\right)\right] \\
\quad-P_{i}+\sum_{r=1, r \neq i}^{N} \beta_{i r}\left(P_{r}-P_{i}\right)<0 .
\end{aligned}
$$

Then, in view of the switching law (15),

$$
\begin{aligned}
& \Delta V(x(k)) \leq \max _{s \in Q} x^{T}(k) \\
& \cdot\left[\left(A_{i}+\Delta A_{i}\right)+\left(B_{i}+\Delta B_{i}\right)\right. \\
&\left.\cdot\left(D_{s} F_{i}+D_{s}^{-} H_{i}\right)\right]^{T} \\
& \cdot P_{i}\left[\left(A_{i}+\Delta A_{i}\right)+\left(B_{i}+\Delta B_{i}\right)\right. \\
&\left.\cdot\left(D_{s} F_{i}+D_{s}^{-} H_{i}\right)\right] x(k)-x^{T}(k) P_{i} x(k) \\
&<-\sum_{r=1, r \neq i}^{N} \beta_{i r} x^{T}(k)\left(P_{r}-P_{i}\right) x(k) \\
& \leq 0,
\end{aligned}
$$

which indicates $\Delta V(x(k))<0$.

Therefore, in view of multiple Lyapunov functions methodology, the switched system (6) is asymptotically stable for all initial states $x_{0} \in \cup_{i=1}^{N}\left(\Omega\left(P_{i}, 1\right) \cap \Phi_{i}\right)$ and admissible uncertainties described by (3) and (4). This completes the proof.

3.2. Controller Design. In this subsection, we study how to design state feedback controllers such that the class of switched system with actuator saturation (6) is robustly stable.

Theorem 5. If there exist positive definite matrices $X_{i}$, matrices $M_{i}, N_{i}$, and a set of scalars $\beta_{i r} \geq 0, \delta_{i r}>0$, and $\lambda_{i}>0$ such that

$$
\left[\begin{array}{ccccccc}
-X_{i}-\sum_{r=1, r \neq i}^{N} \beta_{i r} X_{i} & * & * & * & * & * & * \\
A_{i} X_{i}+B_{i}\left(D_{s} M_{i}+D_{s}^{-} N_{i}\right) & -X_{i} & * & * & * & * & * \\
0 & T_{i}^{T} & -\lambda_{i}^{-1} I & * & * & * & * \\
F_{1 i} X_{i}+F_{2 i}\left(D_{s} M_{i}+D_{s}^{-} N_{i}\right) & 0 & 0 & -\lambda_{i} I & * & * & * \\
X_{i} & 0 & 0 & 0 & -\beta_{i 1}^{-1} X_{1} & * & * \\
X_{i} & 0 & 0 & 0 & 0 & \ddots & * \\
X_{i} & 0 & 0 & 0 & 0 & 0 & -\beta_{i N}^{-1} X_{N}
\end{array}\right]<0,
$$




$$
\left[\begin{array}{ccccc}
X_{i}+\sum_{r=1, r \neq i}^{N} \delta_{i r} X_{i} & * & * & * & * \\
N_{i}^{j} & 1 & * & * & * \\
X_{i} & 0 & \delta_{i 1}^{-1} X_{1} & * & * \\
X_{i} & 0 & 0 & \ddots & * \\
X_{i} & 0 & 0 & 0 & \delta_{i N}^{-1} X_{N}
\end{array}\right] \geq 0, \quad i \in I_{N}, s \in Q, j \in Q_{m}
$$

where $N_{i}^{j}$ denotes the jth row of $N_{i}$, then, under the controllers $F_{i}=M_{i} X_{i}^{-1}$ and the state dependent switching law

$$
\sigma(k)=\operatorname{argmin}\left\{x^{T}(k) X_{i}^{-1} x(k), i \in I_{N}\right\},
$$

the closed-loop switched system (6) with input saturation is robustly asymptotical stable at the origin $\forall x_{0} \in \cup_{i=1}^{N}\left(\Omega\left(X_{i}^{-1}\right.\right.$, 1) $\left.\cap \Phi_{i}\right)$.

Proof. By Lemma 3, (26) is equivalent to

$$
\left[\begin{array}{cccc}
-X_{i}+\sum_{r=1, r \neq i}^{N} \beta_{i r}\left(X_{i} X_{N}^{-1} X_{i}-X_{i}\right) & * & * & * \\
A_{i} X_{i}+B_{i}\left(D_{s} M_{i}+D_{s}^{-} N_{i}\right) & -X_{i} & * & * \\
0 & T_{i}^{T} & -\lambda_{i}^{-1} I & * \\
F_{1 i} X_{i}+F_{2 i}\left(D_{s} M_{i}+D_{s}^{-} N_{i}\right) & 0 & 0 & -\lambda_{i} I
\end{array}\right]<0 .
$$

Let $M_{i}=F_{i} X_{i}, N_{i}=H_{i} X_{i}$, and $X_{i}=P_{i}^{-1}$. Then, preand postmultiplying both sides of inequality (29) by blockdiagonal $\left\{P_{i}, P_{i}, I, I\right\}$, we have

$$
\left[\begin{array}{cccc}
-P_{i}+\sum_{r=1, r \neq i}^{N} \beta_{i r}\left(P_{r}-P_{i}\right) & * & * & * \\
P_{i}\left[A_{i}+B_{i}\left(D_{s} F_{i}+D_{s}^{-} H_{i}\right)\right] & -P_{i} & * & * \\
0 & T_{i}^{T} P_{i} & -\lambda_{i}^{-1} I & * \\
F_{1 i}+F_{2 i}\left(D_{s} F_{i}+D_{s}^{-} H_{i}\right) & 0 & 0 & -\lambda_{i} I
\end{array}\right]<0,
$$

which is exactly (14) in Theorem 4.

Applying a similar method to inequality (27), we can also obtain

$$
\left[\begin{array}{cc}
1 & H_{i}^{j} \\
* & P_{i}-\sum_{r=1, r \neq i}^{N} \delta_{i r}\left(P_{r}-P_{i}\right)
\end{array}\right] \geq 0
$$

where $H_{i}^{j}$ denotes the $j$ th row of $H_{i}$.

Then, we can show that $\Omega\left(P_{i}, 1\right) \cap \Phi_{i} \subset L\left(H_{i}\right)$ is implied by (31). In fact, if we let

$$
G_{i}=P_{i}-\sum_{r=1, r \neq i}^{N} \delta_{i r}\left(P_{r}-P_{i}\right),
$$

since $x^{T} G_{i} x \leq 1$ and $H_{i}^{j} G_{i}^{-1} H_{i}^{j T} \leq 1$, it holds that

$$
2 x^{T} H_{i}^{j T} \leq x^{T} G_{i} x+H_{i}^{j} G_{i}^{-1} H_{i}^{j T} \leq 2 ;
$$

therefore (31) implies $\Omega\left(P_{i}, 1\right) \cap \Phi_{i} \subset L\left(H_{i}\right)$.

Since $P_{i}=X_{i}^{-1}$, the switching rule (28) is the same as (15) of Theorem 4 . Thus, the proof is completed.

Remark 6. When each subsystem of (6) is unstable under actuator saturation, stability of (6) can still be achieved as long as the conditions of Theorem 4 or Theorem 5 are satisfied. This obviously enlarges the scope of systems that can be stabilized subject to actuator saturation.

3.3. Estimation of Domain of Attraction. In this subsection, we will design state feedback controllers and choose $P_{i}$ 's such that the estimated domain of attraction of the closedloop system (6) is maximized with respect to a given shape reference set $X_{R}$.

Let $X_{R} \subset R^{n}$ be a prescribed bounded convex set containing the origin. For a set $\Xi \subset R^{n}$ which contains the origin, define [22]

$$
\alpha_{R}(\Xi):=\sup \left\{\alpha>0: \alpha X_{R} \subset \Xi\right\} .
$$

Obviously, if $\alpha_{R}(\Xi) \geq 1$, then $X_{R} \subset \Xi$. Thus, $\alpha_{R}(\Xi)$ provides a kind of measure of the estimated domain of attraction. Two typical types of $X_{R}$ are the ellipsoid

$$
X_{R}=\left\{x \in R^{n}: x^{T} R x \leq 1, R>0\right\}
$$

and the polyhedron

$$
X_{R}=\operatorname{co}\left\{x_{1}, x_{2}, \ldots, x_{l}\right\},
$$

where $x_{1}, x_{2}, \ldots, x_{l}$ are a priori given points in $R^{n}$.

As a result, the determination of the largest $\cup_{i=1}^{N}\left(\Omega\left(X_{i}^{-1}, 1\right) \cap \Phi_{i}\right)$ inside the domain of attraction can be formulated as the following constrained optimization problem:

$$
\begin{array}{ll}
\sup _{X_{i}, M_{i}, N_{i}, \beta_{i r}, \delta_{i r}, \lambda_{i}} & \alpha, \\
\text { s.t. } & \text { (a) } \alpha X_{R} \subset \Omega\left(X_{i}^{-1}, 1\right), \quad i \in I_{N}, \\
& \text { (b) inequality (26), } \quad i \in I_{N}, s \in Q, \\
& \text { (c) inequality (27), } \quad i \in I_{N}, j \in Q_{m} .
\end{array}
$$



to

Here, we choose $X_{R}$ as an ellipsoid; then (a) is equivalent

$$
\left[\begin{array}{cc}
\frac{1}{\alpha^{2}} R & I \\
I & X_{i}
\end{array}\right] \geq 0
$$

Let $1 / \alpha^{2}=\gamma$; the optimization problem (37) can be rewritten as

$$
\begin{array}{ll}
\inf _{X_{i}, M_{i}, N_{i}, \beta_{i r}, \delta_{i r}, \lambda_{i}} & \gamma, \\
\text { s.t. } & \text { (a) }\left[\begin{array}{cc}
\gamma R & I \\
I & X_{i}
\end{array}\right] \geq 0, \quad i \in I_{N}, \\
& \text { (b) inequality (26), } i \in I_{N}, s \in Q, \\
& \text { (c) inequality (27), } i \in I_{N}, j \in Q_{m} .
\end{array}
$$

Remark 7. If the parameters $\beta_{i r}, \delta_{i r}$, and $\lambda_{i}$ are given in advance, the state feedback controllers and the estimation of domain of attraction are formulated and solved as a set of linear matrix inequality (LMI) optimization problem with respect to other unknown matrix variables.

\section{An Illustrative Example}

In the section, an example is given to illustrate the validity of the results in Section 3.

We consider the following uncertain discrete-time switched linear system subject to actuator saturation:

$$
\begin{aligned}
x(k+1)= & \left(A_{\sigma(k)}+\Delta A_{\sigma(k)}\right) x(k) \\
& +\left(B_{\sigma(k)}+\Delta B_{\sigma(k)}\right) \operatorname{sat}(u(k)),
\end{aligned}
$$

where $\sigma(k) \in I_{2}=\{1,2\}$,

$$
\begin{aligned}
& A_{1}=\left[\begin{array}{cc}
0.2 & 0.1 \\
0 & -1
\end{array}\right], \quad A_{2}=\left[\begin{array}{cc}
-1 & 0 \\
0 & 0.2
\end{array}\right], \\
& B_{1}=\left[\begin{array}{l}
1 \\
0
\end{array}\right], \quad B_{2}=\left[\begin{array}{l}
0 \\
1
\end{array}\right], \quad x(0)=\left[\begin{array}{c}
1 \\
-1
\end{array}\right],
\end{aligned}
$$

the uncertain term $\left[\Delta A_{i}, \Delta B_{i}\right]=T_{i} \Gamma(k)\left[F_{1 i}, F_{2 i}\right]$ with

$$
\begin{aligned}
& T_{1}=\left[\begin{array}{c}
0.1 \\
0
\end{array}\right], \quad T_{2}=\left[\begin{array}{c}
0 \\
0.1
\end{array}\right], \quad F_{11}=\left[\begin{array}{c}
0.15 \\
-0.3
\end{array}\right]^{T}, \\
& F_{12}=\left[\begin{array}{c}
-0.3 \\
0.2
\end{array}\right]^{T}, \\
& F_{12}=-7, \quad F_{22}=-0.2, \quad \Gamma(K)=\sin (k) .
\end{aligned}
$$

The two subsystems are obviously unstable, and it is also easy to see that none of two subsystems subject to actuator saturation can be individually stabilized via state feedback for all admissible uncertainties. Now, we are going to design

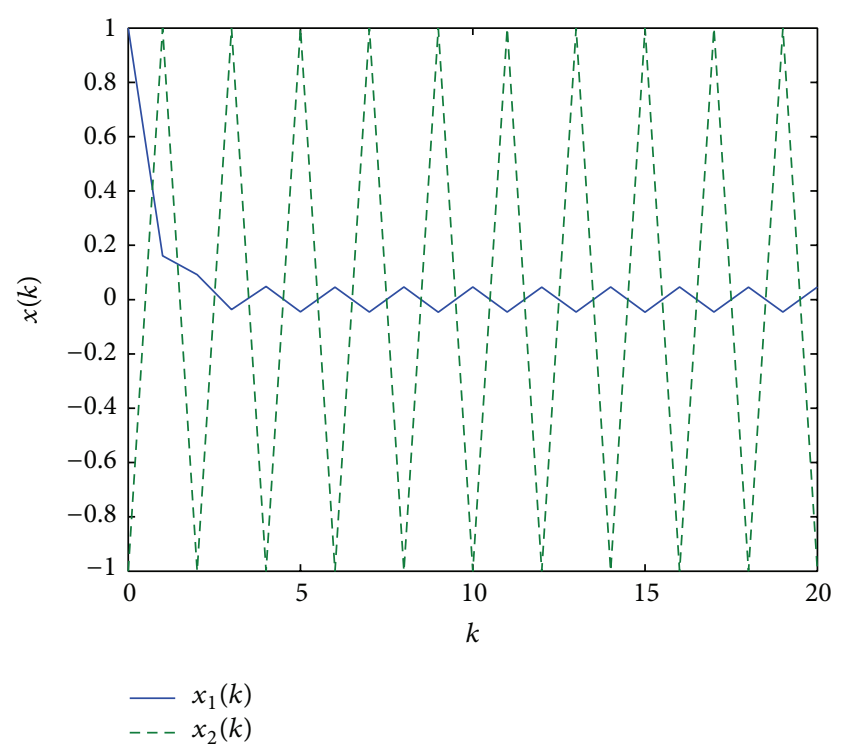

FIgURE 1: The state response of subsystem 1.

a switching law and state feedback controllers to stabilize the switched system (40) with a large domain of attraction.

Let $R=\left[\begin{array}{ll}1 & 0 \\ 0 & 1\end{array}\right], \beta_{1}=\beta_{2}=30, \delta_{1}=\delta_{2}=10$, and $\lambda_{1}=\lambda_{2}=$ 10 . Solving the optimization problem (39) results in

$$
\alpha=18.6842
$$

$$
\begin{aligned}
& X_{1}=\left[\begin{array}{cc}
10.9278 & -0.0026 \\
0.0026 & 10.8016
\end{array}\right], \quad X_{2}=\left[\begin{array}{cc}
10.7819 & 0.0038 \\
0.0038 & 10.9450
\end{array}\right] \text {, } \\
& M_{1}=\left[\begin{array}{c}
0.1890 \\
-0.4744
\end{array}\right]^{T}, \quad M_{2}=\left[\begin{array}{r}
-0.5439 \\
-1.7525
\end{array}\right]^{T} \text {, } \\
& N_{1}=\left[\begin{array}{c}
0.1109 \\
-0.3708
\end{array}\right]^{T}, \quad N_{2}=\left[\begin{array}{c}
-0.3472 \\
0.1630
\end{array}\right]^{T} \text {, } \\
& P_{1}=\left[\begin{array}{ll}
0.0915 & 0.0000 \\
0.0000 & 0.0926
\end{array}\right], \quad P_{2}=\left[\begin{array}{cc}
0.0927 & -0.0000 \\
-0.0000 & 0.0914
\end{array}\right] \text {, } \\
& F_{1}=\left[\begin{array}{c}
0.0173 \\
-0.0439
\end{array}\right]^{T}, \quad F_{2}=\left[\begin{array}{c}
-0.0504 \\
-0.1601
\end{array}\right]^{T} \text {, } \\
& H_{1}=\left[\begin{array}{c}
0.0101 \\
-0.0343
\end{array}\right]^{T}, \quad H_{2}=\left[\begin{array}{c}
-0.0322 \\
0.0149
\end{array}\right]^{T} \text {. }
\end{aligned}
$$

It is easy to verify that none of two controllers makes the associated subsystem stabilizable (see Figures 1 and 2). However, the switched system (40) is stabilizable under the designed switching law together with the state feedback controllers. The state trajectory with the initial state $x(0)=\left[\begin{array}{ll}1-1\end{array}\right]^{T}$ and the input signal are depicted in Figures 3 and 4 , respectively. 


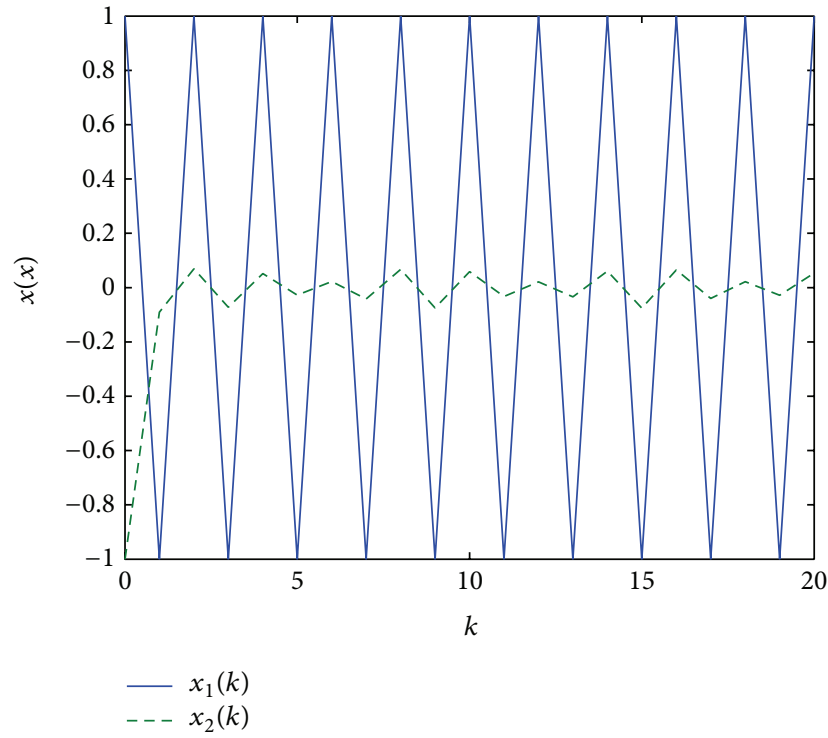

FIGURE 2: The state response of subsystem 2.

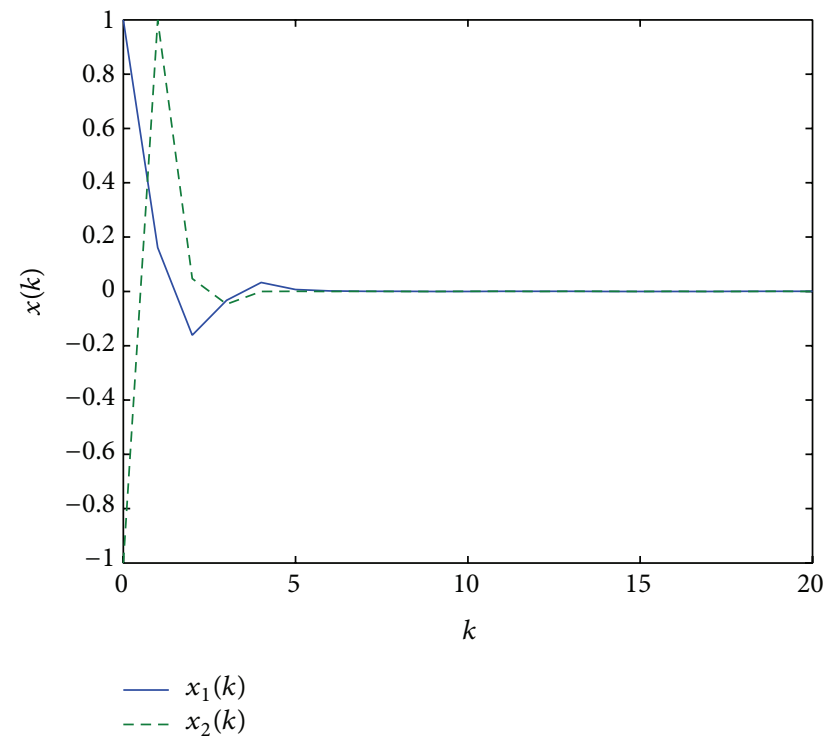

FIGURE 3: The state response of system (40).

In addition, it is worth noticing that if we neglect actuator saturation and design a linear controller, the obtained optimal solutions are

$$
\begin{gathered}
\alpha=10.4807, \\
F_{1}=\left[\begin{array}{c}
0.0088 \\
-0.0421
\end{array}\right]^{T}, \quad F_{2}=\left[\begin{array}{r}
-0.0043 \\
-0.0244
\end{array}\right]^{T},
\end{gathered}
$$

which indicates that, by using the proposed method, the estimation of domain of attraction of the closed loop system is enlarged.

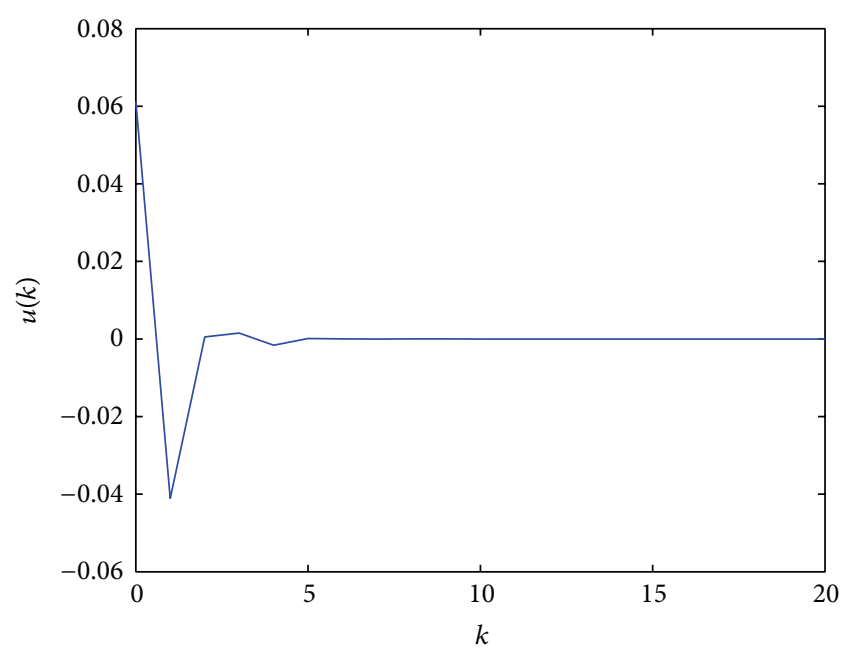

Figure 4: The input signal of system (40).

\section{Conclusions}

This paper has studied the robust stability and robust stabilization for a class of uncertain discrete-time switched linear systems subject to actuator saturation. Via multiple Lyapunov functions approach, sufficient conditions of asymptotic stability have been established. Moreover, if some parameters are selected in advance, the design problem of the robust state feedback control law that maximizes an estimation of the domain of attraction of the considered systems is presented by solving a convex optimization problem with a set of LMI constraints.

It should be noted that due to the complexity of dealing with simultaneous switching and actuator saturation nonlinearity, the proposed method may also cause some conservatism. Thus, how to design a switching law and controllers and to select some parameters to improve the performance of switched systems is a challenging issue which deserves further study in the future.

\section{Conflict of Interests}

The authors declare that there is no conflict of interests regarding the publication of this paper.

\section{Acknowledgments}

This work was supported by the National Natural Science Foundation of China (nos. 61473140 and 61104066) and the Scientific Research Fund of Education Department of Liaoning Province of China (no. L2014159).

\section{References}

[1] D. Liberzon and A. S. Morse, "Basic problems in stability and design of switched systems," IEEE Control Systems Magazine, vol. 19, no. 5, pp. 59-70, 1999.

[2] H. Lin and P. J. Antsaklis, "Stability and stabilizability of switched linear systems: a survey of recent results," IEEE 
Transactions on Automatic Control, vol. 54, no. 2, pp. 308-322, 2009.

[3] Z. D. Sun and S. S. Ge, "Analysis and synthesis of switched linear control systems," Automatica, vol. 41, no. 2, pp. 181-195, 2005.

[4] G. Zhai, B. Hu, K. Yasuda, and A. N. Michel, "Disturbance attenuation properties of time-controlled switched systems," Journal of the Franklin Institute: Engineering and Applied Mathematics, vol. 338, no. 7, pp. 765-779, 2001.

[5] X.-M. Sun, G.-P. Liu, D. Rees, and W. Wang, "Delay-dependent stability for discrete systems with large delay sequence based on switching techniques," Automatica, vol. 44, no. 11, pp. 29022908, 2008.

[6] P. P. Varaiya, "Smart cars on smart roads: problems of control," IEEE Transactions on Automatic Control, vol. 38, no. 2, pp. 195207, 1993.

[7] J. Zhao and M. W. Spong, "Hybrid control for global stabilization of the cart-pendulum system," Automatica, vol. 37, no. 12, pp. 1941-1951, 2001.

[8] W. Zhang, M. S. Branicky, and S. M. Phillips, "Stability of networked control systems," IEEE Control Systems Magazine, vol. 21, no. 1, pp. 84-99, 2001.

[9] X. M. Sun, G. P. Liu, D. Rees, and W. Wang, "Stability of systems with controller failure and time-varying delay," IEEE Transactions on Automatic Control, vol. 53, no. 10, pp. 23912396, 2008.

[10] J. Zhao and G. M. Dimirovski, "Quadratic stability of a class of switched nonlinear systems," IEEE Transactions on Automatic Control, vol. 49, no. 4, pp. 574-578, 2004.

[11] D. Cheng, "Stabilization of planar switched systems," Systems \& Control Letters, vol. 51, no. 2, pp. 79-88, 2004.

[12] D. Cheng, L. Guo, and J. Huang, "On quadratic Lyapunov functions," IEEE Transactions on Automatic Control, vol. 48, no. 5, pp. 885-890, 2003.

[13] J. Daafouz, P. Riedinger, and C. Iung, "Stability analysis and control synthesis for switched systems: a switched Lyapunov function approach," IEEE Transactions on Automatic Control, vol. 47, no. 11, pp. 1883-1887, 2002.

[14] M. S. Branicky, "Multiple Lyapunov functions and other analysis tools for switched and hybrid systems," IEEE Transactions on Automatic Control, vol. 43, no. 4, pp. 475-482, 1998.

[15] S. Pettersson, "Synthesis of switched linear systems," in Proceedings of the 42nd IEEE Conference on Decision and Control, vol. 5, pp. 5283-5288, IEEE, Maui, Hawaii, USA, December 2003.

[16] J. Zhao and D. J. Hill, "On stability, $L_{2}$-gain and $H_{\infty}$ control for switched systems," Automatica, vol. 44, no. 5, pp. 1220-1232, 2008.

[17] J. P. Hespanha and A. S. Morse, "Stability of switched systems with average dwell-time," in Proceedings of the 38th IEEE Conference on Decision and Control (CDC'99), vol. 3, pp. 26552660, Phoenix, Ariz, USA, December 1999.

[18] H. Fang, Z. Lin, and T. Hu, "Analysis of linear systems in the presence of actuator saturation and $\mathscr{L}_{2}$-disturbances," Automatica, vol. 40, no. 7, pp. 1229-1238, 2004.

[19] Q. Zheng and F. Wu, "Output feedback control of saturated discrete-time linear systems using parameter-dependent Lyapunov functions," Systems \& Control Letters, vol. 57, no. 11, pp. 896-903, 2008.

[20] J. M. G. da Silva Jr. and S. Tarbouriech, "Anti-windup design with guaranteed regions of stability for discrete-time linear systems," Systems \& Control Letters, vol. 55, no. 3, pp. 184-192, 2006.
[21] J. M. G. da Silva, D. Limon, T. Alamo, and E. F. Camacho, "Dynamic output feedback for discrete-time systems under amplitude and rate actuator constraints," IEEE Transactions on Automatic Control, vol. 53, no. 10, pp. 2367-2372, 2008.

[22] T. Hu, Z. Lin, and B. M. Chen, "Analysis and design for discretetime linear systems subject to actuator saturation," Systems \& Control Letters, vol. 45, no. 2, pp. 97-112, 2002.

[23] L. Lu and Z. Lin, "Design of switched linear systems in the presence of actuator saturation," IEEE Transactions on Automatic Control, vol. 53, no. 6, pp. 1536-1542, 2008.

[24] L. Lu and Z. Lin, "A switching anti-windup design using multiple Lyapunov functions," IEEE Transactions on Automatic Control, vol. 55, no. 1, pp. 142-148, 2010.

[25] X. Zhang, J. Zhao, G. M. Dimirovski, and R. Ma, "Robust state feedback stabilization of uncertain switched linear systems subject to actuator saturation," in Proceedings of the American Control Conference (ACC '10), pp. 3269-3274, IEEE, Baltimore, Md, USA, June-July 2010.

[26] A. Benzaouia, L. Saydy, and O. Akhrif, "Stability and control synthesis of switched systems subject to actuator saturation," in Proceedings of the American Control Conference (AAC '04), vol. 6, pp. 5818-5823, Boston, Mass, USA, July 2004.

[27] A. Benzaouia, O. Akhrif, and L. Saydy, "Stabilization of switched systems subject to actuator saturation by output feedback," in Proceedings of the 45th IEEE Conference on Decision and Control, pp. 777-782, San Diego, Calif, USA, December 2006.

[28] J. Yu, G. Xie, and L. Wang, "Robust stabilization of discrete-time switched uncertain systems subject to actuator saturation," in Proceedings of the American Control Conference (ACC '07), pp. 2109-2112, New York, NY, USA, July 2007.

[29] I. R. Petersen, "A stabilization algorithm for a class of uncertain linear systems," Systems \& Control Letters, vol. 8, no. 4, pp. 351357, 1987. 


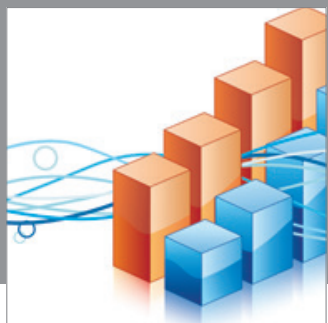

Advances in

Operations Research

mansans

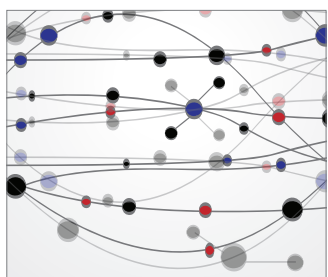

The Scientific World Journal
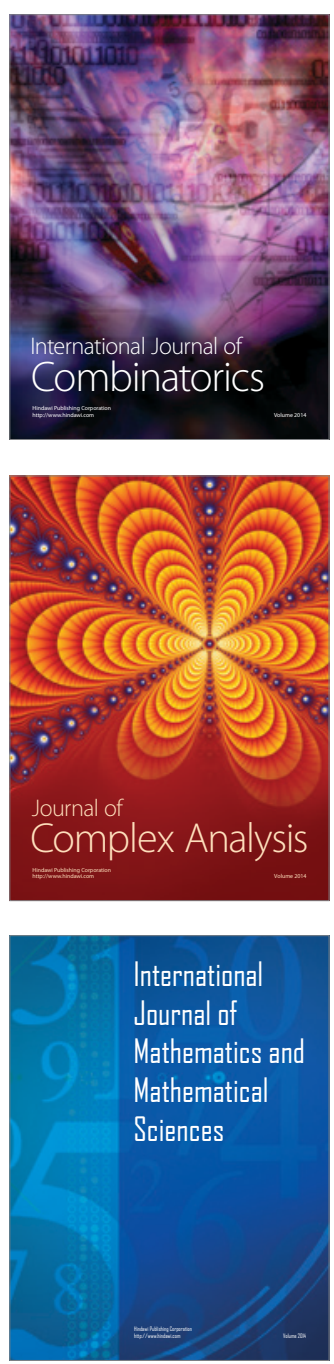
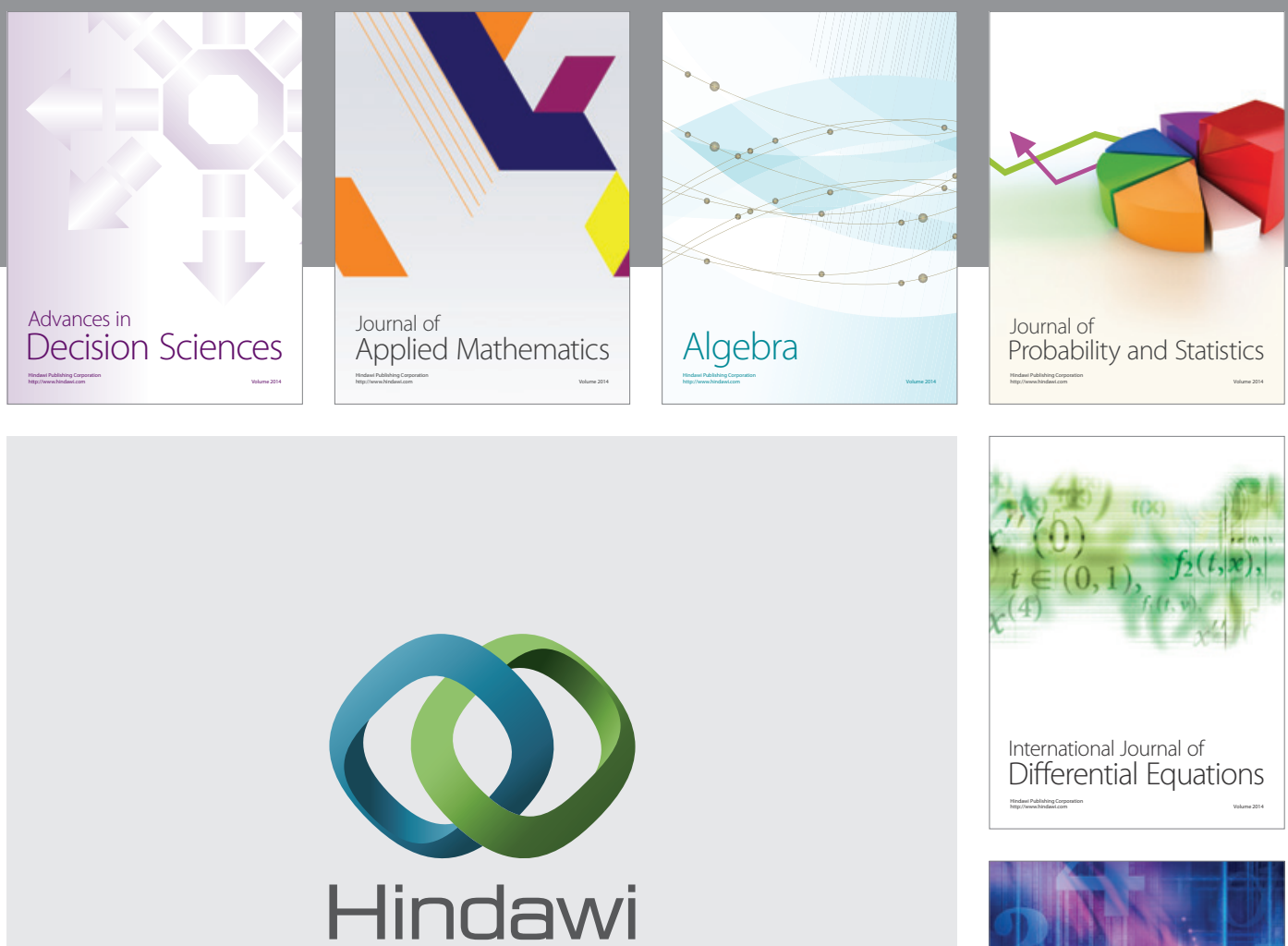

Submit your manuscripts at http://www.hindawi.com
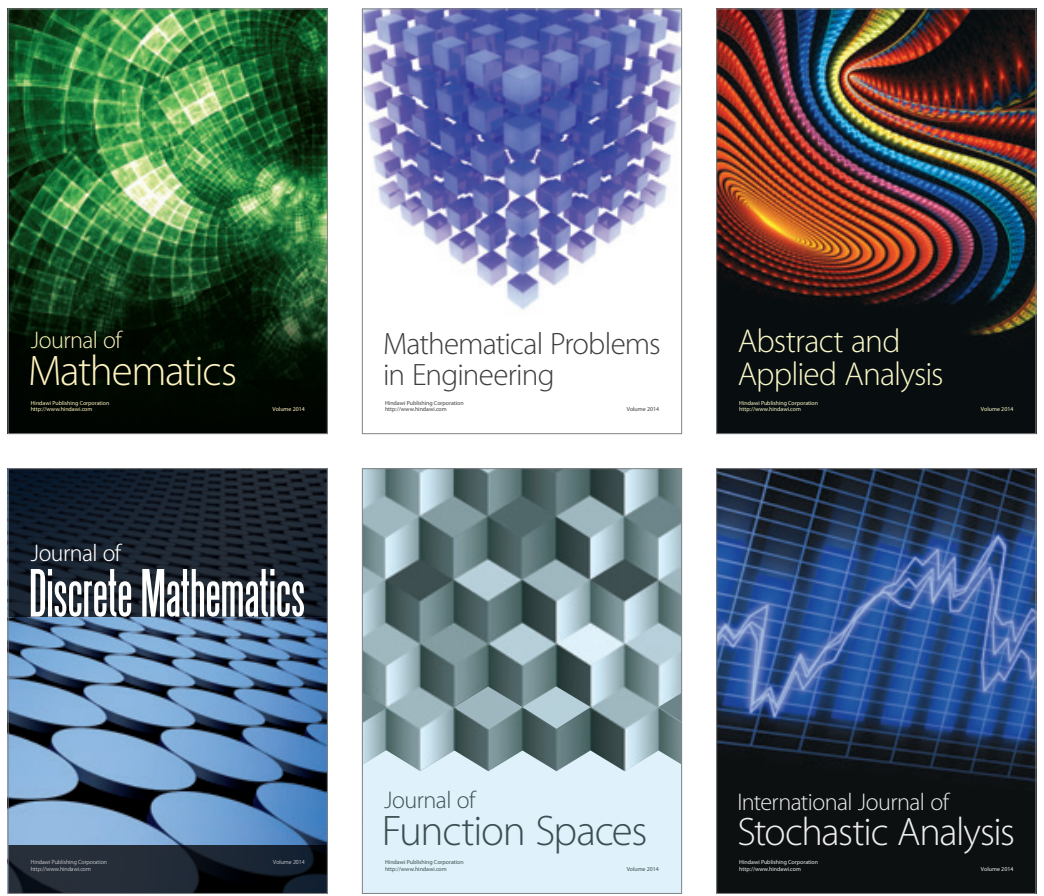

Journal of

Function Spaces

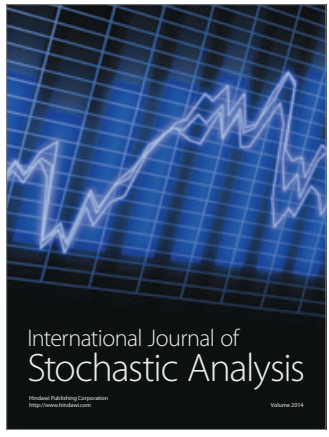

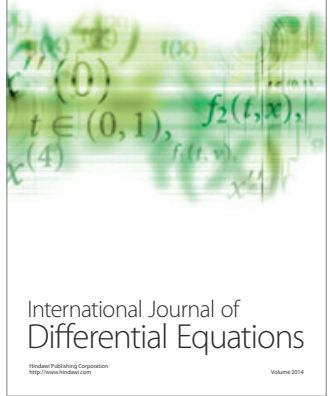
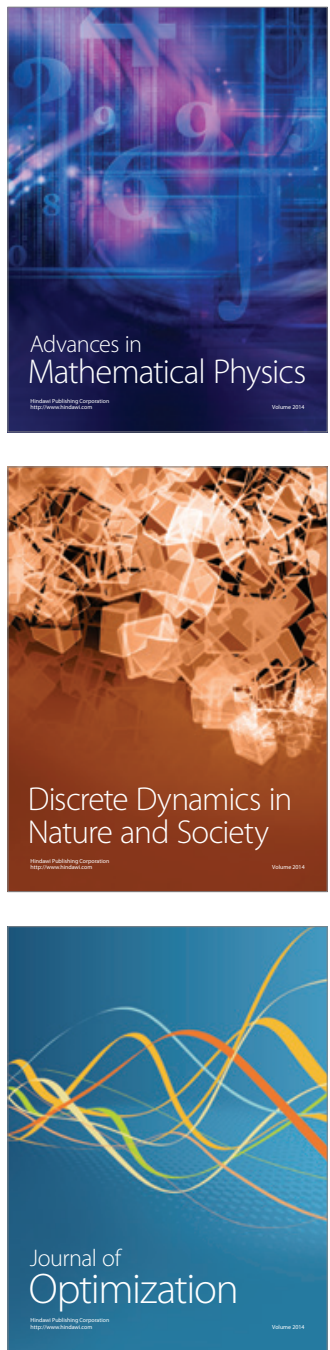